\title{
Reptiles del departamento del Chocó, Colombia
}

\author{
Reptiles of the Department of Chocó, Colombia
}

\author{
Jhon Tailor-Rengifo*, Luis Eladio Rentería-Moreno*
}

\begin{abstract}
Resumen
Por medio de la revisión de literatura científica, trabajos de grado y ejemplares de colección, se presenta la lista anotada de los reptiles del departamento del Chocó con el propósito de generar herramientas y construcción de la línea base del conocimiento del grupo para la región. En la actualidad se conocen alrededor de tres órdenes, 27 familias, 88 géneros y 184 especies; uno de los municipios mejor estudiados en reptiles es Quibdó donde se han realizado numerosos trabajos en diferentes áreas de esta localidad; en la colección de referencia de la fauna chocoana hay ejemplares de 53 especies; los testudinatas son el grupo de reptiles que se encuentra en más peligro, porque aporta un mayor número de especies en alguna de las categorías del CITES y UICN.
\end{abstract}

Palabras clave: Chocó; Reptiles; Línea base; Revisión; Taxonomía.

\begin{abstract}
By means of review of literature, copies of collection and undergrduate, we presents an annotated checklist of reptiles of the chocoana region (Chocó) with the aim of generating tools and a baseline of the knowledge of reptails for the region. At present, three orders, 27 families, 88 genera and 184 speices; among better studied localities in terms of reptiles we have the city of Quibdó where numerous works have been conducted in different areas; in the collection of reference of the fauna chocoana we recorded was 77 species; testudinata was the group of reptiles that they find in someone of the categories of CITES and UICN.
\end{abstract}

Keywords: Chocó; Reptil; Line bases; Review; Taxonomic.

\section{Introducción}

El departamento del Chocó, está considerado como una de las regiones más biodiversas del país; caracterizada como importante área de endemismos, lo que se atribuye a la confluencia de un clima tropical, influido por la existencia de sistemas de montañas, variedad de características hidrológicas, geomorfológicas y a la ubicación geográfica, que lo convierten en un punto intermedio donde se entrelazan las poblaciones de flora y fauna provenientes del norte y sur del continente (Rangel, 1991). Los reptiles constituyen un elemento fundamental de la diversidad de la región chocoana; sin embargo, para el departamento los listados publicados sobre la fauna reptiliana son escasos y los estudios realizados en aras de conocer su distribución, son todavía incipientes, a diferencia de otras regiones del Chocó; estos aspectos dificultan un acercamiento a la verdadera riqueza de reptiles que presenta la región.

\section{Materiales y métodos}

El listado de especies de reptiles presentes en el departamento del Chocó que se presenta en este trabajo, se elaboró tomando como base la revisión de Castaño et al. (2004), en la que incluyeron especímenes del museo de reptiles del Chocó biogeográfico depositados en las siguientes instituciones: Instituto de Ciencias Naturales de la Universi-

* Grupo de Herpetología, Universidad Tecnológica del Chocó, Facultad de Ciencias Básicas, Quibdó, Chocó, Colombia.

e-mail: jhontailorrengifo@hotmail.com

Fecha recepción: Octubre 27, 2010 e-mail: lueremo@gmail.com

Fecha aprobación: Marzo 2, 2011 
Tabla 1. Diversidad de reptiles en el departamento del Chocó.

\begin{tabular}{llccc}
\hline Orden & Suborden & Familia & Géneros & Especies \\
\hline Crocodylidae & & 2 & 2 & 2 \\
Squamata & Amphisbaenia & 1 & 1 & 3 \\
& Sauria & 9 & 24 & 66 \\
& Serpentes & 8 & 51 & 98 \\
Testudinata & & 7 & 10 & 15 \\
Total & & $\mathbf{2 7}$ & $\mathbf{8 8}$ & $\mathbf{1 8 4}$ \\
\hline
\end{tabular}

dad Nacional, Universidad del Valle y museos del exterior; de la presente lista anotada se excluyen las especies cuya información sobre localidad de procedencia o estatus taxonómico no fue clara; Se tuvieron en cuenta los trabajos de investigación, tesis y trabajos de grado realizados en la región, como son: Universidad Tecnológica del Chocó (1996), Rengifo (2002), (Murillo, 2004), García y Moreno (2005), Rentería (2006), Castro-Pérez (2006), Rentería y Rengifo (2006), Hurtado y Ríos (2007), Casa y Gámez (2008), Echavarría y Delgado (2009), Dunlap y Mena (2009), Rengifo (datos no publicados) y Conservación Internacional Colombia (datos no publicados), que han sido la base para muchas de las publicaciones y documentos sobre la fauna de reptiles de departamento del Chocó, como Rengifo et al. (2002, 2003, 2004 y 2007); Murillo et al. (2004a,b y 2006); Murillo (2005a,b); Castro et al. (2006a,b); García et al. (2006); Hurtado et al. (2006); Rentería (2006), (2007); Rentería y Rengifo (2006); Ríos y Hurtado (2007); Rengifo (2007); Rentería et al. (2007), (2009); Dunlap et al. (2009); Echavarría et al. (2009); Gámez et al. (2009); Rengifo y Rentería (2009); Rentería y Rivas (2009).

Además, se revisó el estado de conservación de la herpetofauna del Chocó, consultando en la Lista Roja de la UICN (2008) que menciona las diferentes categorías de amenaza en orden de importancia: En Peligro Crítico (CR), En Peligro (EN), Vulnerable (VU), Casi Amenazada (NT), Preocupación Menor (LC) y Datos Insuficientes (DD). Para la determinación de tráfico ilegal se utilizó, la lista de especies de la Convención sobre el Comercio Internacional de Especies Amenazadas (CITES 2010).

Debido a los múltiples cambios a nivel taxonómico, producto de la intensificación en el trabajo herpetológico en áreas tropicales, la nomenclatura utilizada en el presente trabajo sigue a The TIGR
Reptile Database y la disposición taxonómica para la familia Iguanidae no sigue a Macey et al. (1997) donde se rechaza la división de esta familia; en cuanto a la consideración de las serpientes como un clado de los lagartos (Pough et al. 2001), en este listado se seguirán considerando como grupos separados y al mismo nivel.

\section{Resultados y discusión}

Según la información compilada en este trabajo, para el departamento del Chocó, a la fecha, se han reportado tres órdenes, 27 familias, 88 géneros y 184 especies (Tabla 1, Figura 1), incrementando en 46 especies la lista reportada por Castaño et al. (2004), quienes registran para diferentes puntos de la geografía chocoana alrededor de 138 especies, porque algunas no presentan localidad precisa de captura. El orden Crocodylia está presente con las familias Alligatoridae, Crocodylidae; el Testudinata con las familias Cheloniidae, Chelydridae, Dermochelyidae, Emydidae, Geoemydidae, Kinosternidae y Testudinidae; el Squamata está presente con sus tres subórdenes, el suborden Amphisbenide con la familia Amphisbaenidae, el suborden Sauria o lacertilia con nueve familias (Anguidae, Gekkonidae, Gymnophthalmidae, Polychrotidae, Corytophanidae, Hoplocercidae, Iguanidae, Teiidae y Scincidae), y el Serpentes u ofidios con ocho familias (Anomalepidae, Boidae, Colubridae, Elapidae, Hydrophidae, Leptotyphlopidae, Tropidophidae y Viperidae).

En cuanto a la diversidad de lagartos, el género Anolis (Polychrotidae) con 31 especies es el más representativo de la fauna de reptiles en el departamento del Chocó; también sobresalen los géneros Lepidoblepharis (Gekkonidae), con cinco especies; Ameiva (Teiidae), con tres especies y Basiliscus (Corytophanidae), Diploglosus (Anguidae), 


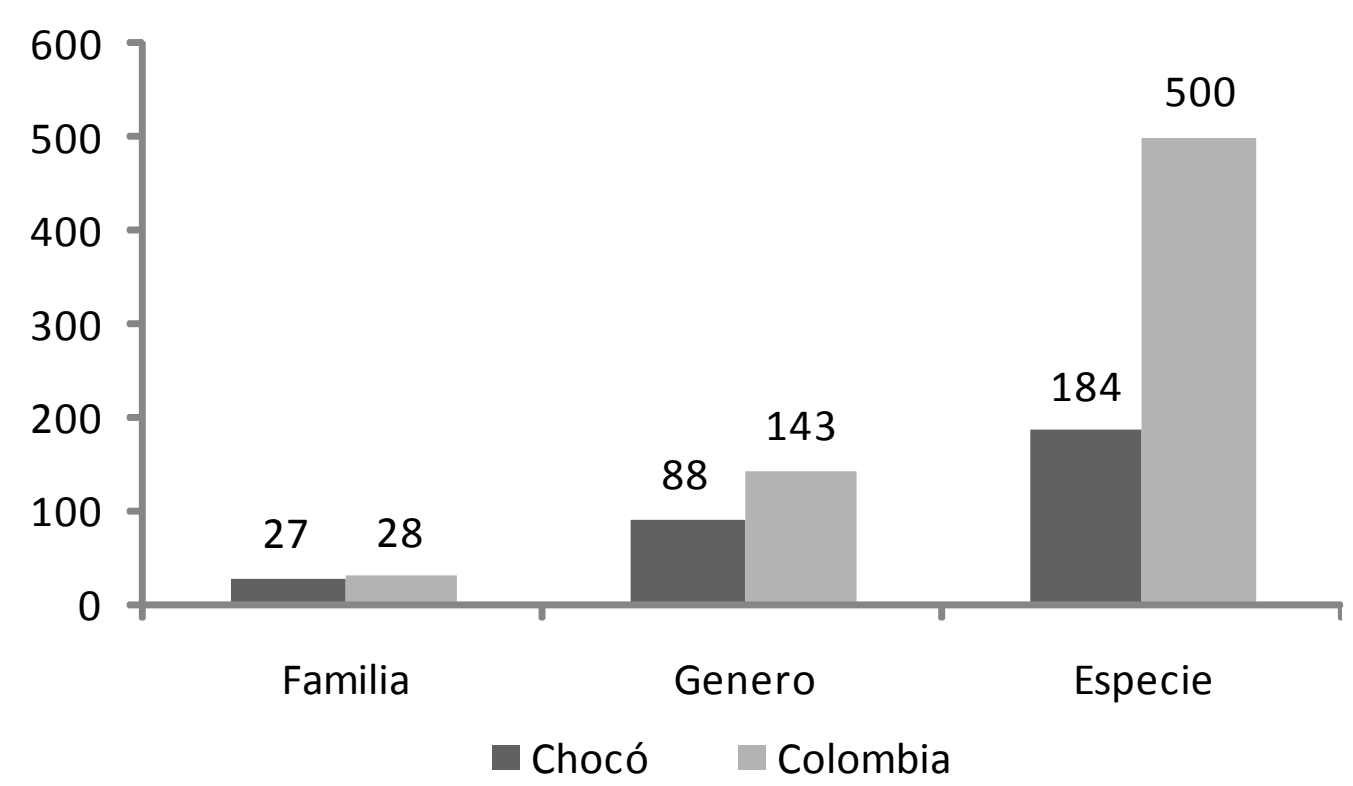

Figura 1. Riqueza de reptiles en el Chocó vs. Colombia.

Gonatodes (Gekkonidae), Cercosauria, Ptychoglossus y Leposoma (Gymnophthalmidae), todos con dos especies cada uno.

En cuanto a la diversidad de serpientes para el departamento del Chocó sobresalen los géneros Atractus (Colubridae) y Micrurus (Elapidae), como los de mayor riqueza específica con ocho especies cada uno, seguidos de Mastigodryas (5) (Colubridae), Corallus (4) (Boidae), Tantilla (4), Dipsas (4), Helicops (4), Chironius (3), Dendrophidium (3), Leptophis (3) y Urotheca (3spp), éstas últimas pertenecientes a la familia Colubridae. Con respecto al grupo de las tortugas, los géneros Kinosternom (Kinosternidae) y Rhinoclemmys (Geoemydidae) sobresalen con tres especies cada uno (Figuras 2, 3).

Según las especies registradas por grupos de reptiles, las serpientes (Squamata: Serpente) fueron el grupo mejor representado con 98 especies (53.3\%), seguido por los lagartos (Squamata: Sauria) con 66 especies $(35.9 \%)$, tortugas (Testudinata) con quince especies (8.2\%), en tanto que los amphisbaenidos y los crocolidianos (Crocodylia) son los grupos menos representados, registrando una baja riqueza (tres y dos especies respectivamente). Se registra a $A$. anchicayae, Anolis purpurescens, Micrurus spurelli, Kinosternum dunni, T. nigra y Tantilla reticulata, como especies endémicas, de las cuales se tiene material de referencia de tres de ellas en la Colección de Referencia de la Fauna Chocoana
(CRFC), sumando así un total de 53 especies en esta colección. Se han registrado especies desde los 0 a $1000 \mathrm{msnm}$, y los chelonios y crocodilios son los grupos de reptiles que se encuentran en alguna de las categorías de amenaza del UICN y el CITES, aunque también se registran algunos saurios y ofidios (Apéndice I). Entre las localidades mejor estudiadas en reptiles está Quibdó donde se han centrado los mayores esfuerzos en el conocimiento de su fauna reptiliana, a través de numerosos trabajos en diferentes áreas de esta localidad.

La información disponible confirma que el departamento del Chocó, se encuentra posicionado entre los de mayor riqueza de reptiles en Colombia y sigue la tendencia nacional donde las serpientes son más representativas que los lagartos, influida por la alta radiación adaptativa que presenta la familia Colubridae en los bosques tropicales.

Todas las especies de reptiles registradas en el UICN y CITES, a excepción de las serpientes, están expuestas a una mayor presión porque se aprovechan como fuente de alimento en las comunidades rurales (afrodecendientes e indígenas), lo que aumenta el riesgo de desaparición local de estas poblaciones, porque no se cuenta con estrategias de conservación y su explotación se realiza de manera indiscriminada.

El área de Quibdó es una de las más estudiadas y reporta la mayor riqueza de reptiles del departa- 


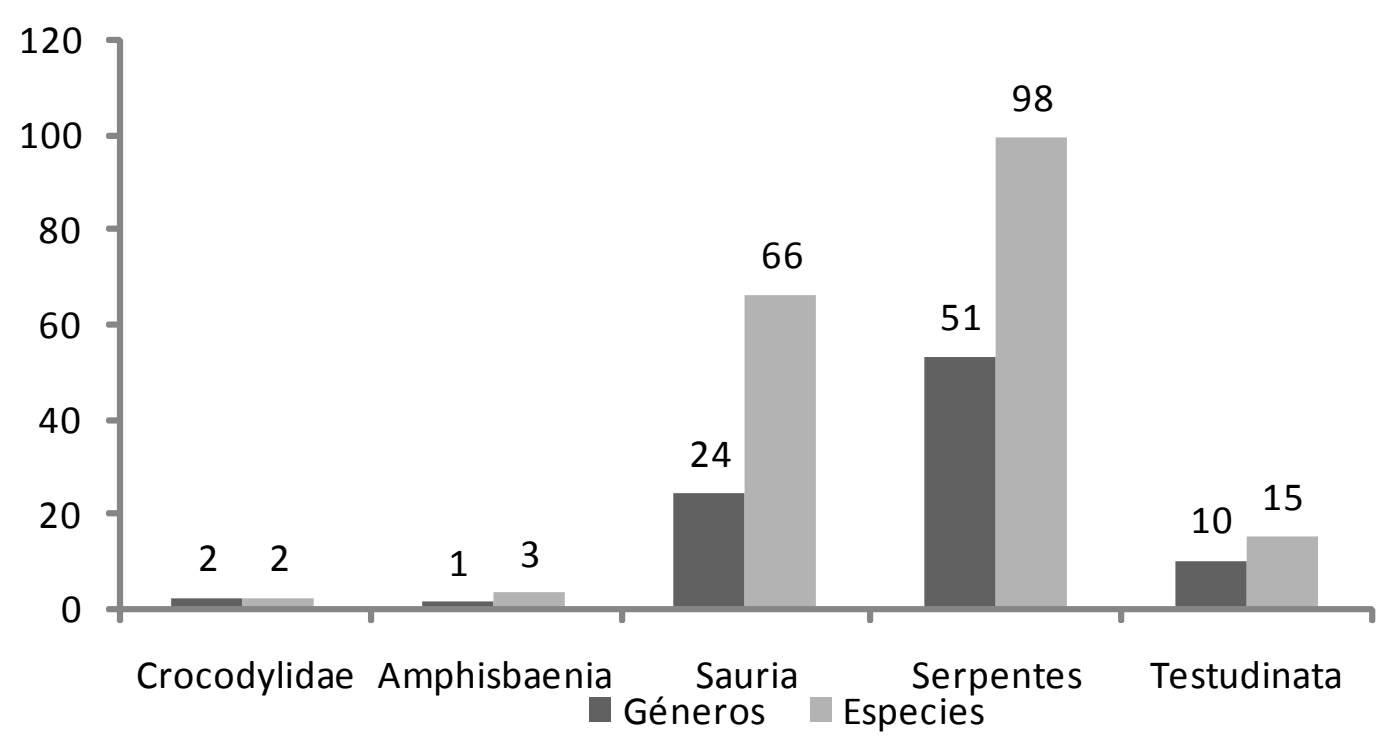

Figura 2. Número de géneros y especies en los diferentes grupos de reptiles en el Chocó.

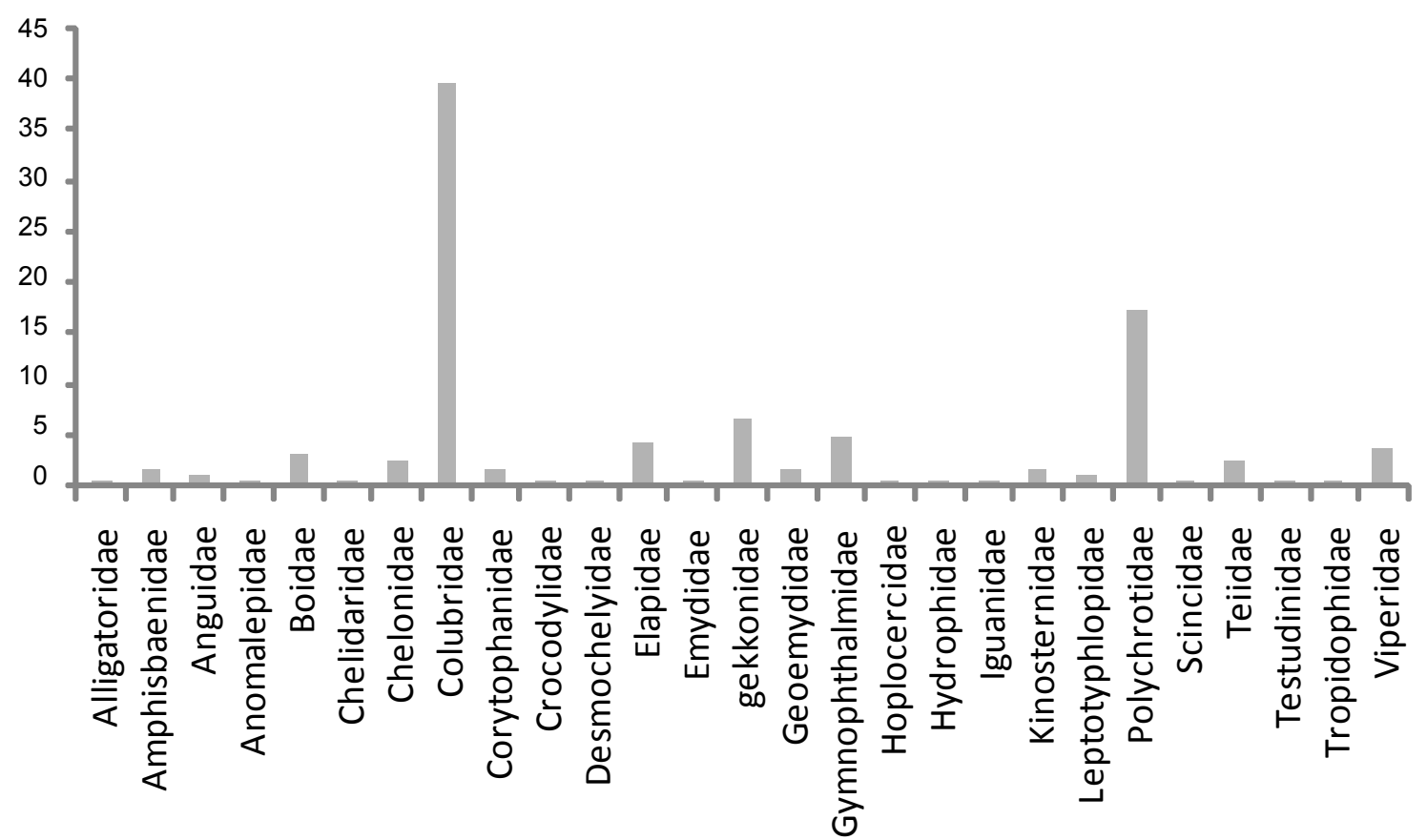

Figura 3. Representatividad porcentual de las familias de reptiles presentes en el Chocó, Colombia.

mento; sin embargo, esto puede ocurrir por la dificultad del acceso para explorar otras áreas. Además, se encuentra ubicada en la zona de la selva pluvial central, que de acuerdo con la subdivisión de la región pacífica sugerida por Rangel-CH (2004), corresponde al centro del Chocó, más exactamente al valle del río Atrato donde se cree, se presenta la mayor concentración de especies de la región.
Por último, el hecho de que en el departamento del Chocó, por distintos factores como falta de vías de acceso, desconocimiento y últimamente orden público, no se puedan estudiar algunas áreas y haya otras localidades inexploradas, permiten discernir que todavía hay muchas especies por descubrir y registrar, y que las cifras consignadas en este estudio van a seguir aumentando. 


\section{Literatura citada}

Castaño O, Cárdenas G, Hernández E, Castro F. 2004. Reptiles en el Chocó biogeográfico. En: Rangel CH (ed.). Colombia, Diversidad Biótica. Tomo IV. Bogotá: Editorial Guadalupe Ltda. p. 277-24.

Castro A, Rengifo J, Murillo F, Echeverri C. 2006a. Caracterización taxonómica y algunos aspectos ecológicos de la comunidad de ofidios en el corregimiento de Salero, Unión Panamericana (Chocó, Colombia). Memorias XLI Congreso Nacional de Ciencias Biológicas, Quibdó. Memorias 11 Congreso Nacional de Zoología, Santa Marta. Panamericana Formas e Impresos S.A.

Castro A, Rengifo J, Murillo F, Asprilla J, Mosquera H, Ríos E. 2006b. Reptiles presentes en la parcela permanente de investigación en biodiversidad en el corregimiento de Salero, municipio de Unión Panamericana, Chocó. Memorias 11 Congreso Nacional de Zoología, Santa Marta. Panamericana Formas e Impresos S.A.

Ceballos F. 2000. Tortugas (Testudinata) marinas y continentales de Colombia. Biota Colomb. 1 (2): 187-94.

Dunlap J, Mena C, Rengifo J, Castro F. 2009. Diversidad de anolis (Lacertilia: Polychrotidae) en áreas con diferentes grados de intervención, corregimiento de Salero, Unión Panamericana, Chocó. En: Rengifo J, Ramos Y. (eds.). Memorias. Primer Congreso Regional de Herpetología. Quibdó: Universidad Tecnológica del Chocó.

Echavarría J, Delgado J, Rengifo J, Rentería L. 2009. Caracterización espacio-temporal de la comunidad de serpientes presentes en el corregimiento de Samurindó, municipio del Atrato, Chocó, Colombia. En: Rengifo J, Ramos Y. (eds.). Memorias. Primer Congreso Regional de Herpetología. Quibdó: Universidad Tecnológica del Chocó.

Gámez K, Casas A, Murillo F, Rentería L. 2009. Estudio preliminar de las serpientes del Medio Atrato. Chocó, Colombia. En: Rengifo J, Ramos Y. (eds.). Memorias. Primer Congreso Regional de Herpetología. Quibdó: Universidad Tecnológica del Chocó.

García U, Rengifo J, Moreno F, Jiménez A. 2006. Diversidad de lagartos (Squamata: Lacertilia) en el bosque de cuatro localidades asociadas a la cuenca del río Cabí, Chocó, Colombia. Revista Institucional. Universidad Tecnológica del Chocó DLC. 25 (2): 47-55.

Hurtado C, Ríos E, Rengifo J. 2006. Reptiles presentes en dos zonas de bosque del Chocó biogeográfico colombiano. Memorias 11 Congreso Nacional de Zoología, Santa Marta. Panamericana Formas e Impresos S.A.

Murillo F. 2005a. Nuevos reportes de ofidios (Squamata: Serpentes) para el departamento del Chocó. Revista Institucional. Universidad Tecnológica del Chocó DLC. 22 (2): 37-44.

Murillo F, Mauricio A, Rengifo J, Asprilla J. 2004b. Primeros registros de ofidios (Suborden: Serpentes) en la estación ambiental de Tutunendo, Chocó (Colombia). Bioetnia. 1 (1): 15-9.

Murillo F, Moreno E, Roa Y, Mena Y, Rengifo J. 2004a. Caracterización ecológica de la ofidiofauna en el corre- gimiento de Pacurita, Chocó, Colombia. Revista Científica de la Universidad Tecnológica del Chocó DLC 19: 45-9.

Murill F, Rivas T, Saldarriaga M. 2006. Serpientes de la cuenca del río Cabí, Chocó biogeográfico colombiano. Memorias 11 Congreso Nacional de Zoología, Santa Marta. Panamericana Formas e Impresos S.A.

Rengifo J, García U, Moreno F. 2007. Distribución vertical y por sustrato de saurios presentes en la cuenca del río Cabi. Chocó, Colombia. En: Memorias XLII Congreso Nacional de Ciencias Biológicas, Barranquilla.

Rengifo F, Asprilla J, Jiménez A, Rengifo J. Castro A. 2002. Ecología y estructura de la comunidad de reptiles presentes en el corregimiento de Pacurita, municipio de Quibdó, Chocó, Colombia. V Seminario Internacional del Medio Ambiente y Desarrollo Sostenible, 2002, Bucaramanga, Santander. División editorial y publicaciones, Universidad Industrial de Santander, p. 45-52.

Rengifo J. 2007. Diversidad de reptiles presentes en tres unidades paisajísticas del tramo Afirmado-Nuquí, departamento del Chocó, Colombia. En: Memorias XLII Congreso Nacional de Ciencias Biológicas, Barranquilla.

Rengifo J, Rentería E. 2009. Reptiles de la región natural chocoana, estado del arte. En: Rengifo J, Ramos Y. (eds.). Memorias. Primer Congreso Regional de Herpetología. Quibdó: Universidad Tecnológica del Chocó.

Rengifo J, Jiménez A, Asprilla J, Rengifo J, Roa Y, Moreno F. 2003. Distribución vertical y por sustrato de reptiles en un bosque pluvial tropical (bp-T) del Chocó. Revista Institucional de la Universidad Tecnológica del Chocó DLC. 18 (2): 43-9.

Rengifo J, Asprilla J, Rengifo J, Jiménez A, Roa Y. 2004. Una aproximación a la herpetofauna (anfibios y reptiles) del municipio de Nóvita, departamento del Chocó, Colombia. Revista Institucional de la Universidad Tecnológica del Chocó DLC. 20 (1): 39-44.

Rentería L, Rengifo J, Moya J. 2007. Comunidad de reptiles presente en el sotobosque de la selva pluvial central del departamento del Chocó. Revista Institucional Universidad Tecnológica del Chocó: Investigación, Biodiversidad y Desarrollo. 26 (2): 23-36.

Rentería L, Rengifo J. 2006. Revisión taxonómica del género Anolis (Squamata, Lacertilia: Polychrotidae) para el departamento del Chocó. En: Memorias XLI Congreso Nacional de Ciencias Biológicas. Quibdó: Asociación Colombiana de Ciencias Biológicas.

Rentería L. 2006. Caracterización taxonómica de la comunidad de reptiles presente en el sotobosque de la estación ambiental Tutunendo, Quibdó, Chocó. Tesis de grado. Quibdó: Facultad de Ciencias, Universidad Tecnológica del Chocó DLC.

Rentería L. 2007. Caracterización de la comunidad de reptiles presentes en el municipio de río Quito. Informe Institucional Universidad Tecnológica del Chocó.

Rentería L, Rivas T. 2009. Etnozoología y aspectos ecológicos de la ofidiofauna asociada con cultivos agrícolas presentes en la selva pluvial central del municipio de Quibdó, Chocó. En: Rengifo J, Ramos Y. (eds.). Memorias. Primer Congreso Regional de Herpetología. Quibdó: Universidad 
Tecnológica del Chocó.

Rentería L, Rivas T, Bonilla N. 2009. Taxonomía y aspectos ecológicos de la comunidad de reptiles en áreas con diferente tipo de intervención en la cabecera municipal de Quibdó, Chocó. En: Rengifo J, Ramos Y. (eds.). Memorias. Primer Congreso Regional de Herpetología. Quibdó: Universidad Tecnológica del Chocó.

Ríos E, Hurtado C. 2007. Contribución al conocimiento de la ecología de las comunidades de lagartos presentes en dos zonas de bosque en el Chocó biogeográfico colombiano. Tesis de grado. Chocó: Facultad de Ciencias, Universidad Tecnológica del Chocó DLC.
Universidad Tecnológica del Chocó, OREWA, Fundación Neotrópicos e Instituto Nacional de Vías. 1996. Estudio de impacto ambiental, social y cultural. Proyecto vial: río Pató-rió Baudó-Tribugá. Bogotá: Universidad Tecnológica del Chocó.

Urbina U, Londoño M, García G. 2006. Diversidad de la ofidiofauna en cuatro áreas con diferente grado de perturbación antropogénica en el parque nacional natural isla Gorgona, Pacífico colombiano. Memorias 11 Congreso Nacional de Zoología, Santa Marta. 
Apéndice I

Listado taxonómico

\begin{tabular}{|c|c|c|c|c|}
\hline Taxon & Altura & $\begin{array}{l}\text { Estatus de } \\
\text { amenaza }\end{array}$ & $\begin{array}{l}\text { Colección } \\
\text { UTCh }\end{array}$ & Fuentes de registro \\
\hline \multicolumn{5}{|l|}{$\begin{array}{l}\text { Orden Crocodylia } \\
\text { Familia Alligatoridae }\end{array}$} \\
\hline Caiman crocodilus (Linnaeus, 1758) & $0-1000$ & Cites II & $x$ & $\begin{array}{l}\text { Rengifo (2002) y } \\
\text { Renteria (2006) }\end{array}$ \\
\hline \multicolumn{5}{|l|}{ Familia Crocodylidae } \\
\hline Crocodylus acutus (Cuvier, 1807) & $0-100$ & VU, Cites I & & Castaño et al. (2004) \\
\hline $\begin{array}{l}\text { Oden Testudinata } \\
\text { Familia Cheloniidae }\end{array}$ & \multicolumn{4}{|c|}{ Familia Cheloniidae } \\
\hline Caretta caretta (Linnaeus, 1758) & 0 & EN, Cites I & & Castaño et al. (2004) \\
\hline Chelonia agassizii (Bacourt, 1868) & 0 & EN, Cites I & & Castaño et al. (2004) \\
\hline Chelonia mydas (Linnaeus, 1758) & & EN, Cites I & & Ceballos (2000) \\
\hline Eretmochelys imbricata (Linnaeus, 1766) & 0 & CR, Cites I & & Castaño et al. (2004) \\
\hline Lepidochelys olivacea (Eschscholtz, 1829) & & VU, Cites I & & Castaño et al. (2004) \\
\hline \multicolumn{5}{|l|}{ Familia Chelydridae } \\
\hline Chelydra serpentina (Linnaeus, 1758) & $0-1200$ & & $x$ & Castaño et al. (2004) \\
\hline \multicolumn{5}{|l|}{ Familia Dermochelydae } \\
\hline Dermochelys coriacea (Linnaeus, 1766) & 0 & CR, Cites I & & Castaño et al. (2004) \\
\hline \multicolumn{5}{|l|}{ Familia Emydidae } \\
\hline Trachemys scripta (Thunberg, 1792) & $0-1000$ & VU & $\mathrm{x}$ & Castaño et al. (2004) \\
\hline \multicolumn{5}{|l|}{ Familia Geoemydidae } \\
\hline Rhinoclemmys annulata (Gray, 1860) & $0-200$ & LR/NT & & Castaño et al. (2004) \\
\hline Rhinoclemmys melanosterna (Gray, 1861) & $0-50$ & & & Castaño et al. (2004) \\
\hline Rhinoclemmys nasuta (Boulenger, 1902) & $0-50$ & LR/NT & & Rentería et al (2008) \\
\hline \multicolumn{5}{|l|}{ Familia Kinosternidae } \\
\hline Kinosternon dunni (Schmidt, 1947) & $0-100$ & VU & $\mathrm{x}$ & Castaño et al. (2004) \\
\hline Kinosternon leucostomum (Cope 1887) & $0-100$ & & & $\begin{array}{l}\text { Castaño et al. (2004) } \\
\text { Renteria (2007) }\end{array}$ \\
\hline Kinosternon scorpioides (Linnaeus, 1766) & $0-100$ & VU & $\mathrm{x}$ & $\begin{array}{l}\text { Ceballos }(2000) \\
\text { Rengifo et al. 2002, } \\
\text { Rentería } 2008\end{array}$ \\
\hline \multicolumn{5}{|l|}{ Familia Testudinidae } \\
\hline Geochelone carbonaria (Spix, 1824) & $0-50$ & Cites II & & Castaño et al. (2004) \\
\hline \multicolumn{5}{|l|}{$\begin{array}{l}\text { Orden Squamata } \\
\text { Suborden Amphisbaenia } \\
\text { Familia Amphisbaenidae }\end{array}$} \\
\hline Amphisbaena alba (Linnaeus, 1758) & $100-500$ & & & $\begin{array}{l}\text { Esqueda \& La Marca, } \\
1999\end{array}$ \\
\hline Amphisbaena fuliginosa (Linnaeus, 1758) & $0-100$ & & & Castaño et al. (2004) \\
\hline Amphisbaena spurrelli (Bolenger, 1915) & $0-100$ & & & Castaño et al. (2004) \\
\hline \multicolumn{5}{|l|}{$\begin{array}{l}\text { Suborden Sauria } \\
\text { Familia Anguidae }\end{array}$} \\
\hline Diploglosus monotropis (Kuhl, 1820) & $0-100$ & & $\mathrm{x}$ & $\begin{array}{l}\text { Castaño et al. (2004) } \\
\text { Rengifo et al., (2003) }\end{array}$ \\
\hline $\begin{array}{l}\text { Diploglosus montisilvestris (Myers, 1973) } \\
\text { Familia Gekkonidae }\end{array}$ & $500-1500$ & & & Castaño et al. (2004) \\
\hline Gonatodes albogularis (Dumeril \& Bibron, 1836) & $0-1500$ & & $\mathrm{x}$ & $\begin{array}{l}\text { Castaño et al. (2004) } \\
\text { Rengifo et al. (2003) }\end{array}$ \\
\hline Gonatodes caudiscutatus (Günther, 1859) & & & & Swash \& Still 2000 \\
\hline Hemidactylus brookii (Gray, 1845) & $0-1000$ & & $\mathrm{x}$ & $\begin{array}{l}\text { Renteria et al. (2008) } \\
\text { Rengifo et al. (2003) }\end{array}$ \\
\hline Lepidoblepharis intermedius (Boulenger, 1914) & $0-500$ & & & Castaño et al. (2004) \\
\hline Lepidoblepharis microlepis (Noble, 1923) & $0-100$ & & $\mathrm{x}$ & Renteria et al. (2008) \\
\hline Lepidoblepharis peraccae (Boulenger, 1908) & $0-640$ & & & Castaño et al. (2004) \\
\hline Lepidoblepharis ruthveni (Parker, 1926) & & & & \\
\hline Lepidoblepharis sanctaemartae (Ruthven, 1916) & & & & Castaño et al. (2004) \\
\hline Lepidodactylus lugubris (Dumeril \& Bibron 1836) & $0-800$ & & $\mathrm{x}$ & García et al (2006) \\
\hline Pseudogonatodes perivianus (Huey \& Dixon 1970) & $0-100$ & & $\mathrm{x}$ & García et al (2006) \\
\hline $\begin{array}{l}\text { Sphaerodactylus lineolatus (Lichtensitein \& } \\
\text { von Martens 1856) }\end{array}$ & $0-50$ & & & Castaño et al. (2004) \\
\hline
\end{tabular}


Apéndice I

Listado taxonómico (continuación)

\begin{tabular}{|c|c|c|c|c|}
\hline Taxon & Altura & $\begin{array}{l}\text { Estatus de } \\
\text { amenaza }\end{array}$ & $\begin{array}{l}\text { Colección } \\
\text { UTCh }\end{array}$ & Fuentes de registro \\
\hline Thecadactylus rapicauda (Houttuyn, 1782) & $50-1200$ & & $\mathrm{x}$ & Castaño et al. (2004) \\
\hline \multicolumn{5}{|l|}{ Familia Gymnophthalmidae } \\
\hline Anadia vittata Boulenger, 1913 & $20-800$ & & & Castaño et al. (2004) \\
\hline Bachia pallidiceps (Cope, 1862) & $0-50$ & & & Castaño et al. (2004) \\
\hline Echinosauria horrida Boulenger, 1890 & $0-1500$ & & $x$ & Castaño et al. (2004) \\
\hline Leposoma rugiceps (Cope, 1869) & $0-100$ & & $\mathrm{x}$ & $\begin{array}{l}\text { García et al. (2006) } \\
\text { Rengifo (2002) }\end{array}$ \\
\hline Leposoma southi (Ruthven \& Gaige, 1924) & $0-1000$ & & $\mathrm{x}$ & Castaño et al. (2004) \\
\hline Cercosauria argulus (Peter, 1862) & 0 & & & Castaño et al. (2004) \\
\hline Cercosauria vertebralis (O'Shaughnessy, 1879) & 750 & & & Castaño et al. (2004) \\
\hline Ptychoglossus festae (Peracca, 1896) & Sin Localidad & & & Castaño et al. (2004) \\
\hline Ptychoglossus plicatus (Taylor, 1949) & Sin Localidad & & & Castaño et al. (2004) \\
\hline Familia Polychrotidae & & & & Castaño et al. (2004) \\
\hline Anolis anchicayae Poe, Velasco, Miyata \& Williams, 2009 & $0-1000$ & & $\mathrm{x}$ & Castaño et al. (2004) \\
\hline Anolis antioquiae Williams, 1985 & $1500-2300$ & & & \\
\hline Anolis antonii Boulenger, 1908 & $800-2000$ & & & \\
\hline Anolis auratus (Daudin, 1802) & $0-1400$ & & & Castaño et al. (2004) \\
\hline Anolis binotatus Peter, 1863 & $500-800$ & & & Castaño et al. (2004) \\
\hline Anolis biporcatus (Wiliams 1966) & $70-500$ & & $\mathrm{x}$ & Castaño et al. (2004) \\
\hline Anolis chloris (Boulenger, 1898) & $0-800$ & & $\mathrm{x}$ & Castaño et al. (2004) \\
\hline Anolis chocorum (Wiliams and Duellman,1967) & $20-1100$ & & $\mathrm{x}$ & Castaño et al. (2004) \\
\hline Anolis frenatus (Cope, 1899) & $20-1300$ & & & Castaño et al. (2004) \\
\hline Anolis gracilipes (Boulenger, 1898) & $20-800$ & & & Castaño et al. (2004) \\
\hline Anolis granuliceps (Boulenger, 1898) & $30-1500$ & & $\mathrm{x}$ & Castaño et al. (2004) \\
\hline Anolis latifrons (Berthold, 1846) & $60-600$ & & $\mathrm{x}$ & Castaño et al. (2004) \\
\hline Anolis lemurinus Cope, 1861 & $80-880$ & & & \\
\hline Anolis lyra Poe, Velasco, Miyata \& Williams, 2009 & $0-400$ & & $\mathrm{x}$ & $\begin{array}{l}\text { Castaño et al. (2004) } \\
\text { Rengifo (no publicado) }\end{array}$ \\
\hline Anolis macrolepis (Boulenger, 1911) & $150-300$ & & & Castaño et al. (2004) \\
\hline Anolis maculigula Williams, 1984 & $600-2000$ & & & Castaño et al. (2004) \\
\hline Anolis maculiventris (Boulenger, 1898) & $0-800$ & & $\mathrm{x}$ & Castaño et al. (2004) \\
\hline Anolis malkini Cope, 1862 & & & $\mathrm{x}$ & $\begin{array}{l}\text { Castaño et al. (2004) } \\
\text { Rengifo (no publicado) }\end{array}$ \\
\hline Anolis mirus (Williams, 1963) & $100-1500$ & & & Castaño et al. (2004) \\
\hline Anolis notopholis (Boulenger, 1896) & $0-1200$ & & $\mathrm{x}$ & Castaño et al. (2004) \\
\hline Anolis pentaprion (Cope, 1862) & $30-500$ & & & Castaño et al. (2004) \\
\hline Anolis peraccae (Boulenger, 1898) & $0-700$ & & $\mathrm{x}$ & Castaño et al. (2004) \\
\hline Anolis poecilopus (Cope, 1862) & $100-1500$ & & & Castaño et al. (2004) \\
\hline Anolis princeps Boulenger, 1902 & $0-1000$ & & & Williams (1988) \\
\hline Anolis purpurescens (Cope, 1899) & $80-350$ & & & Castaño et al. (2004) \\
\hline Anolis radulinus Cope, 1862 & $400-1800$ & & & Günther (1885) \\
\hline Anolis rivalis Williams, 1984 & $200-1300$ & & $\mathrm{x}$ & Castaño et al. (2004) \\
\hline Anolis tropidogaster (Hallowell, 1857) & $0-1500$ & & & Castaño et al. (2004) \\
\hline Anolis ventrimaculatus Boulenger, 1911 & $1200-2500$ & & & Ayala (1986) \\
\hline Anolis vicarius Williams, 1986 & $0-800$ & & & Castaño et al. (2004) \\
\hline Anolis vittigerus Cope, 1862 & $0-500$ & & $\mathrm{x}$ & Castaño et al. (2004) \\
\hline \multicolumn{5}{|l|}{ Familia Corytophanidae } \\
\hline Basiliscus basiliscus Linnaeus, 1758 & $0-200$ & & $x$ & Castaño et al. (2004) \\
\hline Basiliscus galeritus Dumeril, 1851 & $0-1000$ & & $x$ & Castaño et al. (2004) \\
\hline Corytophanes cristatus Merrem, 1821 & $0-800$ & & $x$ & Castaño et al. (2004) \\
\hline \multicolumn{5}{|l|}{ Familia Haplocercidae } \\
\hline Enyalioides heterolepis Bocourt, 1885 & $0-1000$ & & $x$ & Castaño et al. (2004) \\
\hline \multicolumn{5}{|l|}{ Familia Iguanidae } \\
\hline Iguana iguana Linnaeus, 1817 & $0-1500$ & Cites II & & Castaño et al. (2004) \\
\hline \multicolumn{5}{|l|}{ Familia Scincidae } \\
\hline Mabuya mabuya (Lecépede, 1788) & $0-1800$ & & $x$ & Castaño et al. (2004) \\
\hline \multicolumn{5}{|l|}{ Familia Teiidae } \\
\hline Ameiva anomala Echternacht, 1977 & $0-800$ & & $\mathrm{x}$ & Castaño et al. (2004) \\
\hline
\end{tabular}


Apéndice I

Listado taxonómico (continuación)

\begin{tabular}{|c|c|c|c|c|}
\hline Taxon & Altura & $\begin{array}{l}\text { Estatus de } \\
\text { amenaza }\end{array}$ & $\begin{array}{l}\text { Colección } \\
\text { UTCh }\end{array}$ & Fuentes de registro \\
\hline Ameiva festiva (Lichtenstein \& von Martens 1856) & $100-1000$ & & $\mathrm{x}$ & Castaño et al. (2004) \\
\hline Ameiva leptophrys Cope, 1893 & $0-800$ & & & Castaño et al. (2004) \\
\hline Cnemidophorus lemniscatus (Linnaeus, 1758) & $0-1000$ & & & Castaño et al. (2004) \\
\hline $\begin{array}{l}\text { Tupinambis teguixin (Linnaeus, } 1758 \\
\text { Suborden Serpentes } \\
\text { Familia Anomalepidae }\end{array}$ & $0-100$ & Cites II & & Castaño et al. (2004) \\
\hline Helminthophis flavoterminatus Peter, 1857 & $100-800$ & & & Castaño et al. (2004) \\
\hline \multicolumn{5}{|l|}{ Familia Boidae } \\
\hline Boa constrictor Linnaeus, 1758 & $0-1000$ & Cites II & $\mathrm{x}$ & Castaño et al. (2004) \\
\hline Corallus annulatus (Cope 1876) & $0-250$ & Cites II & $\mathrm{x}$ & Castaño et al. (2004) \\
\hline Corallus caninus Linnaeus, 1758 & $80-1000$ & Cites II & & Castaño et al. (2004) \\
\hline Corallus hortalanus (Hederson1997) & $80-1000$ & Cites II & $\mathrm{x}$ & Murillo et al. (2003) \\
\hline Corallus ruschenbergerii Cope, 1876 & $30-600$ & Cites II & & Castaño et al. (2004) \\
\hline $\begin{array}{l}\text { Epicrates cenchria Linnaeus, } 1758 \\
\text { Familia Colubridae }\end{array}$ & $50-1000$ & Cites II & $\mathrm{x}$ & Castaño et al. (2004) \\
\hline Amastridium veliferum Cope, 1860 & 1610 & & & \\
\hline Atractus clarki Dunn \& Bailey, 1939 & $80-400$ & & & Castaño et al. (2004) \\
\hline Atractus elaps (Gúnther, 1858) & 100 & & $\mathrm{x}$ & Castro-Pérez (2006) \\
\hline Atractus iridescens Peracca, 1896 & 70 & & & Castaño et al. (2004) \\
\hline Atractus loveridgei Amaral, 1930 & 750 & & & Castaño et al. (2004) \\
\hline Atractus major Boulenger, 1894 & 100 & & $x$ & Renteria et al. (2008) \\
\hline Atractus multicintactus (Jan, 1865) & $40-350$ & & & Castaño et al. (2004) \\
\hline Atractus nicefori Amaral, 1930 & $1500-2500$ & & & \\
\hline Atractus obstrusirostri Werner, 1916 & 100 & & $x$ & Castro-Pérez (2006) \\
\hline Chironius carinatus (Linnaeus, 1758) & $20-400$ & & & Castaño et al. (2004) \\
\hline Chironius grandiscamis (Peters, 1869) & $300-800$ & & & Castaño et al. (2004) \\
\hline Chironius fuscus Linnaeus, 1758 & $150-650$ & & & \\
\hline Clelia clelia (Daudin, 1758) & $20-1750$ & Cites II & $x$ & Castaño et al. (2004) \\
\hline Dendrophidion bivittatus Dumeril, Bibron \& Dumeril, 1918 & $0-500$ & & $x$ & Castaño et al. (2004) \\
\hline Dendrophidion dendrophis Shlegel, 1837 & $100-1200$ & & & Castaño et al. (2004) \\
\hline Dendrophidion pecarinatus (Cope, 1893) & $0-1200$ & & $x$ & Castaño et al. (2004) \\
\hline Diaphorolepis wagneri Jan, 1863 & $100-1600$ & & & \\
\hline Dipsas gracilis Boulenger, 1902 & $30-800$ & & & Castaño et al. (2004) \\
\hline Dipsas sanctijoannis Boulenger, 1911 & $0-1500$ & & & Castaño et al. (2004) \\
\hline Dipsas temporalis (Werner, 1909) & $0-1000$ & & $x$ & Castaño et al. (2004) \\
\hline Dipsas variegata (Dumeril \& Bibron 1918) & 100 & & $x$ & Murillo (2005) \\
\hline Drymarchon corais (Boie, 1827) & $100-800$ & & & Castaño et al. (2004) \\
\hline Drymobius rhombifer (Gúnther, 1860) & $30-1600$ & & & Castaño et al. (2004) \\
\hline Enulius sclateri (Boulenger, 1894) & $0-1300$ & & & Castaño et al. (2004) \\
\hline Erythrolamprus aesculapii (Linnaeus, 1766) & $50-1400$ & & & Castaño et al. (2004) \\
\hline Erythrolamprus mimus (Cope, 1868) & $0-1200$ & & & Castaño et al. (2004) \\
\hline Geophis brachycephalus (Cope, 1871) & $0-800$ & & & Castaño et al. (2004) \\
\hline Helicops angulatus (Linnaeus 1758) & 100 & & $\mathrm{x}$ & Murillo (2005) \\
\hline Helicops danieli Amaral, 1938 & 43 & & & Castaño et al. (2004) \\
\hline Helicops scalaris Jan, 1865 & $80-350$ & & & \\
\hline Helicops leopardinus (Schlegel 1837) & 100 & & & Murillo et al (2006) \\
\hline Imantodes cenchoa (Linnaeus, 1758) & $20-1800$ & & $\mathrm{x}$ & Castaño et al. (2004) \\
\hline Imantodes inornatus (Boulenger, 1876) & $30-1000$ & & $\mathrm{x}$ & Castaño et al. (2004) \\
\hline Lampropeltis triangulum (Lacepede, 1789) & $1400-2000$ & & & Castaño et al. (2004) \\
\hline Leptodeira annulata (Linnaeus, 1758) & $100-1200$ & & & Castaño et al. (2004) \\
\hline Leptodeira septentrionalis (Kennicott, 1859) & $40-1300$ & & $\mathrm{x}$ & Castaño et al. (2004) \\
\hline Leptophis ahaetulla (Linnaeus, 1758) & $0-750$ & & $\mathrm{x}$ & Castaño et al. (2004) \\
\hline Leptophis depressirostris (Cope, 1871) & $20-600$ & & & Castaño et al. (2004) \\
\hline Leptophis riveti Despax, 1910 & $60-1600$ & & & Castaño et al. (2004) \\
\hline Liophis epinephelus Cope, 1862 & $80-1500$ & & & Castaño et al. (2004) \\
\hline Masticophis mentovarius Duméril, Bibron \& Duméril, 1854 & $40-400$ & & & \\
\hline Mastigodryas boddaerti (Sentzen, 1796) & $50-2000$ & & & Castaño et al. (2004) \\
\hline Mastigodryas danieli Amaral, 1935 & $300-2400$ & & & Castaño et al. (2004) \\
\hline Mastigodryas melanolomus (Cope 1868) & 100 & & $\mathrm{x}$ & Murillo (2005) \\
\hline
\end{tabular}


Apéndice I

Listado taxonómico (continuación)

\begin{tabular}{|c|c|c|c|c|}
\hline Taxon & Altura & $\begin{array}{c}\text { Estatus de } \\
\text { amenaza }\end{array}$ & $\begin{array}{l}\text { Colección } \\
\text { UTCh }\end{array}$ & Fuentes de registro \\
\hline Mastigodryas pleei Duméril, Bibron \& Duméril, 1854 & $20-1200$ & & & $\begin{array}{l}\text { Conservacion Interna- } \\
\text { cional (datos no publ.) }\end{array}$ \\
\hline Mastigodryas pulchriceps (Cope 1868 & 100 & & $x$ & Murillo (2005) \\
\hline Ninia atrata (Hallowell, 1845 ) & $0-750$ & & $\mathrm{x}$ & Castaño et al. (2004) \\
\hline Nothopsis rugosus Cope, 1871 & $100-1000$ & & $\mathrm{x}$ & Castaño et al. (2004) \\
\hline Oxybelis aeneus (Wagler, 1824) & $20-1400$ & & $\mathrm{x}$ & Castaño et al. (2004) \\
\hline Oxybelis brevirostris (Cope, 1861) & $0-600$ & & $\mathrm{x}$ & Castaño et al. (2004) \\
\hline Oxyrhopus petola (Linnaeus, 1758) & 100 & & $\mathrm{x}$ & Castaño et al. (2004) \\
\hline Phimophis guianensis (Troschel, 1848) & $0-400$ & & & Castaño et al. (2004) \\
\hline Pliocercus euryzonus Cope, 1862 & $0-800$ & & & Castaño et al. (2004) \\
\hline Pseustes poecilonotus (Günther, 1858) & $20-1350$ & & $x$ & Castaño et al. (2004) \\
\hline Pseustes shropshirei (Barbour \& Amaral, 1924 ) & $0-1300$ & & & Castaño et al. (2004) \\
\hline Rhadinaea decorata (Günther, 1858) & $500-800$ & & & Castaño et al. (2004) \\
\hline Rhinobothyum bovallii Anderson, 1916 & 100 & & $\mathrm{x}$ & $\begin{array}{l}\text { Renteria et al. (2008) } \\
\text { Rengifo et al. } 2002\end{array}$ \\
\hline Sibon argus (Cope, 1876) & 100 & & $\mathrm{x}$ & Castro-Perez (2006) \\
\hline Sibon nebulata (Linnaeus, 1758) & $0-1750$ & & & Castaño et al (2004) \\
\hline Siphlophis cervinus (Laurenti, 1768) & $0-150$ & & & Castaño et al. (2004) \\
\hline Siphlophis compressus (Daudin, 1803) & $0-100$ & & & Castaño et al. (2004) \\
\hline Spilotes pullatus (Linnaeus, 1758) & $0-1700$ & & $\mathrm{x}$ & Castaño et al. (2004) \\
\hline Stenorrhina degenhardtii (Berthold, 1854) & $80-1850$ & & $x$ & Castaño et al. (2004) \\
\hline Tantilla alticola (Boulenger,1903) & $100-1800$ & & & Castaño et al. (2004) \\
\hline Tantilla melanocephala (Linnaeus, 1758) & $20-1200$ & & $\mathrm{x}$ & Castaño et al. (2004) \\
\hline Tantilla nigra (Boulenger, 1914) & $50-500$ & & & Castaño et al. (2004) \\
\hline Tantilla reticulata Cope, 1860 & $50-500$ & & & Castaño et al. (2004) \\
\hline Tretanorhinus taeniatus Boulenger, 1903 & $0-100$ & & & Castaño et al. (2004) \\
\hline Urotheca fulviceps (Cope, 1886) & $40-450$ & & & Castaño et al. (2004) \\
\hline Urotheca lateristriga (Berthold 1859) & $100-2000$ & & & Castaño et al. (2004) \\
\hline Urotheca pachyura Cope, 1875 & 100 & & $\mathrm{x}$ & $\begin{array}{l}\text { Renteria et al. (2008) } \\
\text { Rengifo et al. } 2002\end{array}$ \\
\hline $\begin{array}{l}\text { Xenodon rabdocephalus (Wied, 1824) } \\
\text { Familia Elapidae }\end{array}$ & $0-500$ & & & Castaño et al. (2004) \\
\hline Micrurus ancoralis (Jan, 1872) & $0-600$ & & $x$ & Castaño et al. (2004) \\
\hline Micrurus bocourti Jan, 1872 & $500-1500$ & & & \\
\hline Micrurus clarki Schmidt, 1936 & 700 & & & Castaño et al. (2004) \\
\hline Micrurus dissoleucus (Cope, 1859) & 65 & & & Castaño et al. (2004) \\
\hline Micrurus dumerilii (Jan, 1958) & $0-600$ & & $\mathrm{x}$ & Castaño et al. (2004) \\
\hline Micrurus mipartitus (Dumeril, Bibron \& Dumeril 1854) & $0-2750$ & & & Castaño et al. (2004) \\
\hline Micrurus multicutatus (Rendahl \& Vestergren 1940) & 100 & & $\mathrm{x}$ & Murillo (2003) \\
\hline $\begin{array}{l}\text { Micrurus spurrelli ( Boulenger, 1914) } \\
\text { Familia Hydrophiidae }\end{array}$ & 70 & & & Castaño et al. (2004) \\
\hline Pelamis platurus (Linnaeus, 1769) & 0 & & & Castaño et al. (2004) \\
\hline Familia Leptotyphlopidae & & & & \\
\hline Epictia goudotii (Dumeril \& Bibron, 1844) & 100 & & $\mathrm{x}$ & Castro-Pérez (2006) \\
\hline Tricheilostoma macrolepis (Peter, 1857) & 100 & & $x$ & Murillo et al. (2006) \\
\hline Familia Tropidophiidae & & & & \\
\hline Trachyboa boulengeri Peracca, 1910 & $70-805$ & Cites II & & Castaño et al. (2004) \\
\hline Familia Viperidae & & & & \\
\hline Bothriechis schlegelii (Berthold, 1846) & $0-2650$ & & $\mathrm{x}$ & Castaño et al. (2004) \\
\hline Bothriopsis punctata (Garcia, 1896) & $0-750$ & & $\mathrm{x}$ & Castaño et al. (2004) \\
\hline Bothrocophias myersi (Gutberlet \& Campbell, 2001) & $0-200$ & & $\mathrm{x}$ & \\
\hline Bothrops asper (Garman, 1883) & $0-1600$ & & $\mathrm{x}$ & Castaño et al. (2004) \\
\hline Lachesis achrocorda (Garcia, 1896) & $0-300$ & & $\mathrm{x}$ & Castaño et al. (2004) \\
\hline Porthidium lansbergii (Schlegel, 1841) & $0-1200$ & & & Castaño et al. (2004) \\
\hline Porthidium nasutum (Bocourt, 1968) & $0-100$ & & $x$ & Castaño et al. (2004) \\
\hline
\end{tabular}

